Musées, Patrimoine et Culture scientifiques et techniques

$133 \mid 2011$

janvier - février 2011

\title{
L'itinérance : une seconde vie pour les expositions
}

\section{Olivier Soichot}

\section{OpenEdition}

Journals

Édition électronique

URL : http://journals.openedition.org/ocim/667

DOI : $10.4000 /$ ocim.667

ISSN : 2108-646X

Éditeur

OCIM

Édition imprimée

Date de publication : 1 janvier 2011

Pagination : 32-36

ISSN : 0994-1908

Référence électronique

Olivier Soichot, "L'itinérance : une seconde vie pour les expositions », La Lettre de l'OCIM [En ligne],

133 | 2011, mis en ligne le 01 janvier 2013, consulté le 01 mai 2019. URL : http://

journals.openedition.org/ocim/667; DOI : 10.4000/ocim.667

Ce document a été généré automatiquement le 1 mai 2019.

Tous droits réservés 


\title{
L'itinérance : une seconde vie pour les expositions
}

\author{
Olivier Soichot
}

La Cité de l'Espace a été créée en 1997. Depuis quand et pourquoi a-t-elle décidé de s'investir dans le domaine des expositions itinérantes?

1 Cela s'est fait naturellement. La Cité de l'Espace est productrice de toutes ses expositions temporaires qui sont généralement présentées pendant un an dans le pavillon des expositions. Il apparaissait donc intéressant de donner une seconde vie à ces productions en les faisant itinérer vers d'autres établissements culturels dans le cadre de leur programmation temporaire.

Toutes les expositions temporaires conçues par la Cité de l'Espace sont donc au départ pensées pour l'itinérance?

2 En effet, le service des expositions itinérantes rentre dans le processus de création des expositions temporaires en veillant à ce que son cahier des charges soit respecté. La complexité réside cependant dans le fait de concevoir des expositions qui répondent à un double objectif : celui de coller à la stratégie de la Cité de l'Espace en terme de thème, d'immersion et d'intégration au lieu mais également aux différentes et nombreuses contraintes logistiques d'itinérances. C'est la raison pour laquelle, devant la complexité de la tâche, nous avons décidé de segmenter le process de création en deux temps. Seule, une partie du cahier des charges "itinérance" est intégrée au premier concept de création. Après sa présentation à la Cité l'Espace et avant sa mise en itinérance, l'exposition fait l'objet de modifications conséquentes où la totalité de notre cahier des charges est alors intégré.

À travers son service d'expositions itinérantes, quels sont les objectifs poursuivis par la Cité de l'Espace?

Le service des expositions itinérantes est une petite entreprise intégrée dont la vocation est de proposer un véritable service « clé en main » et de qualité à tous les musées. C'est bien évidemment un moyen de promotion de la Cité de l'Espace mais c'est avant tout une manière pour elle de développer, hors les murs, sa mission de diffusion de la culture 
scientifique en accomplissant son savoir-faire en termes de médiation et d'ingénierie culturelle.

Comment évolue la part de ce secteur depuis sa création?

4 Au fil des années, l'offre des expositions itinérantes se régénère avec de nouvelles thématiques. Aujourd'hui, nous réalisons une dizaine de projets par an. L'offre s'étoffe et la question de l'itinérance des expositions fait plus que jamais partie intégrante du processus de création des services muséologiques de la Cité de l'Espace.

En quelques mots, comment caractériseriez-vous cette offre proposée par la Cité de I'Espace?

5 L'offre en termes de contenus et le service associé que nous proposons autour de la mise à disposition d'expositions forment un "package » indissociable et relativement attractif pour les institutions muséales. Le service, la réactivité, la capacité à proposer des produits associés aux expositions sont des points forts qui ont pour objectif de simplifier considérablement le travail des lieux d'accueil dans le cadre de la programmation d'une exposition. Nous tendons vers une logique qui n'a que pour seul objectif d'être à l'écoute de nos clients en leur offrant le meilleur service.

Qu'est-ce qui vous différencie d'autres producteurs diffuseurs d'expositions itinérantes? Peut-on dire qu'il existe une marque de fabrique propre à la Cité de l'Espace au niveau de son offre?

6 Nous avons été les premiers à proposer avec nos expositions itinérantes des spectacles associés afin que les musées bénéficient d'une prestation d'animation périphérique « prêt à l'emploi » pour leurs visiteurs. Toutes nos expositions itinérantes sont aujourd'hui proposées avec ce dispositif d'animation/spectacles ${ }^{1}$. Il s'agit là d'une véritable valeur ajoutée aux expositions et d'un phénoménal gain de temps pour les services de médiation des musées programmant l'exposition.

7 Côté communication, un kit est proposé avec la mise à disposition du visuel de l'exposition et l'intervention d'un invité "spécial inauguration». La Cité de l'Espace dispose en effet, grâce à son excellente insertion dans le tissu scientifique et industriel, d'un réseau de partenaires scientifiques susceptibles d'être sollicités en fonction du thème de l'exposition.

8 D'une manière générale, notre capacité à élaborer des prestations clé en main, notre réactivité et notre connaissance des visiteurs est un atout que nos clients apprécient. On pourrait dire que c'est notre marque de fabrique !

Quels types de structures louent vos expositions?

9 Nous sommes sollicités par des interlocuteurs extrêmement variés ce qui nous oblige à être à l'écoute de fonctionnements et d'objectifs différents, ce qui est véritablement passionnant.

10 En général, il s'agit de musées (nationaux et internationaux), de centres de cultures scientifiques, de parcs thématiques, de centres culturels, de collectivités comme des conseils généraux, mairies, offices de tourisme mais nous travaillons également avec des espaces à fort trafic comme des aéroports, des palais des congrès, des sièges d'entreprises et autres espaces commerciaux.

11 Concernant les musées et notamment les internationaux, nous remarquons un intérêt croissant des musées d'Histoire naturelle pour des expositions temporaires radicalement différentes de leur cœur de thématique. Étonner les visiteurs et créer plus de contraste 
dans les programmations semble être une nouvelle tendance pour ces musées traditionnellement «à collections ». Il s'agit également de capter l'attention des visiteurs en répondant aux nouvelles tendances de "consommation de savoir» en perpétuelle mutation afin de générer des recettes de fréquentation indispensables à l'équilibre financier de l'établissement.

Justement, pour quels types de publics sont en priorité conçues vos productions?

Fidèle au positionnement ludo-éducatif de la Cité de l'Espace, nos expositions s'adressent à toute la famille. Le spectacle associé concerne généralement un plus jeune public et offre une prestation conviviale basée sur l'échange et la connaissance avec un contenu plus doux.

On le voit également, votre rayon d'action touche beaucoup l'international. Comment se passe la diffusion de vos productions à l'étranger? Par quels canaux passez-vous pour diffuser votre offre?

13 Notre zone de diffusion géographique concerne en effet l'étranger pour $80 \%$. Depuis 12 ans, nous avons ainsi établi au fil des années de solides relations avec les grandes institutions muséales et leurs différents réseaux. Nous adhérons par exemple à celui d'ECSITE, très impliqué dans la diffusion de la culture scientifique à travers le monde. Le réseau des services culturels d'ambassades françaises à l'étranger est également sollicité.

Enfin, nous travaillons avec un réseau d'agences événementielles spécialisées. Mais le meilleur canal de diffusion reste le bouche à oreille, c'est la raison pour laquelle nos prestations, tant sur le contenu que sur l'aspect organisationnel, doivent être irréprochables.

Au-delà du bouche-à-oreille, comment communiquez-vous sur vos expositions ?

Nous répondons à une logique marketing avec la mise en place d'actions de promotion récurrentes. Les médias sont également un excellent levier à la promotion de nos expositions dans le cadre de l'inauguration d'une exposition où à travers la publication d'articles d'actualité.

Comment qualifieriez-vous l'état actuel du marché des expositions itinérantes ?

16 Il est très concurrentiel et nous devons faire preuve en permanence d'imagination et de réactivité pour répondre à la demande des musées dont les budgets sont de plus en plus réduits.

Quel impact la crise économique a-t-elle précisément sur votre activité ?

17 Il est bien évident que les restrictions budgétaires ont touché de plein fouet les institutions culturelles. Nous restons cependant confiants. Les chiffres parlent d'eux mêmes, nous n'avons accusé aucune baisse significative depuis 2008. Nous restons proches de nos clients et ils continuent à nous faire confiance.

Quelles grandes évolutions sur ce marché avez-vous constaté depuis que vous menez cette activité à la Cité de l'Espace?

18 «L'émotionnel » et "le sociétal » sont des angles de traitement de thématiques qui sont de plus en plus demandés. Il ne s'agit plus aujourd'hui de diffuser un contenu sérieux de manière brut, il faut travailler la manière d'interpeller le visiteur pour le faire rentrer dans le thème et donc dans le processus de découverte. C'est une évolution des méthodes de médiations : on ne peut plus espérer intéresser une personne à un sujet si on ne se connecte pas préalablement à son niveau de référence sur le thème et à ses centres d'intérêts. 
Comment financez-vous vos expositions itinérantes? demande très forte.

21 Pour moi, il s'agit surtout de la bonne prise en compte de notre cahier des charges : la modularité, la qualité du contenu, l'acheminement et la fiabilité. Nous accordons également une grande importance au conditionnement de l'exposition étant donné que nous avons beaucoup de matériel fragile (audiovisuel, maquettes, mobilier...) Nous utilisons des flight case - particulièrement utiles lors de transport par voie maritime ou par avion - pour garantir une parfaite sécurité des objets et manipes.

Le choix des thèmes des expositions est également une étape primordiale dans le processus de création. Comment se passe cette phase d'élaboration à la Cité de l'Espace?

La direction marketing de la Cité de l'Espace intervient bien évidemment dans le choix des thèmes. Au final, ce choix s'appuie sur trois facteurs récurrents: l'actualité, les attentes du public, et la prise en compte des différents retours des institutions muséales, dans le cadre de la mise à disposition d'expositions itinérantes.

23 Aujourd'hui, nous disposons de cinq expositions itinérantes: Destination Mars ; InvenTerre, regards sur un vaisseau planétaire ; Cosmomania ; Expérience Lune et Extraterrestre (qui est actuellement présentée à la Cité de l'Espace). Une prochaine exposition viendra compléter notre offre : De la Terre aux étoiles. Proposée à la location dès avril 2011, elle sera composée de six espaces thématiques et d'un laboratoire à expériences.

Quelles sont les principales contraintes que vous rencontrez dans la conception d'une exposition?

Il s'agit principalement de contraintes de modularité. Concevoir une grande exposition qui s'adapte à tous les types de lieux alliant chacun des contraintes architecturales propres, c'est souvent un véritable casse-tête pour mes collègues muséographes et architectes!

Combien de personnes l'activité d'expositions itinérantes mobilise-t-elle au sein de la Cité de l'Espace? temps plein. Pour le reste de l'équipe, il s'agit de sous-traitants.

Pour les opérations de montage et de démontage, nous faisons appel à une équipe de prestataires extérieurs spécialisée dans le secteur de l'audiovisuel et des décors. Pour le volet animation, les animateurs et formateurs sont détachés ponctuellement de la Cité de l'Espace.

27 Enfin, coté production, c'est la direction des programmes de la Cité de l'Espace qui s'attache à créer les expositions. C'est un grand Département où cohabitent muséographes et architectes. Deux enseignants détachés interviennent également sur le volet pédagogique de l'exposition. 
Travaillez-vous avec des partenaires extérieurs pour la phase de conception/réalisation? intéressant. Nous pouvons mettre en scène des éléments surprenants comme le HRV, le système optique du satellite SPOT, ou encore de véritables objets spatiaux ayant volé dans la station Mir.

Que devient une exposition une fois sa vie terminée? leur satisfaction et de celle de leurs publics. Nous avons l'avantage de proposer des expositions qui ont déjà été testées par 300000 visiteurs à la Cité de l'Espace pendant au minimum un an. Cette période "test » offre une garantie de qualité aux institutions d'accueil. La Cité de l'Espace dispose d'un observatoire des publics où chaque commentaire de visiteur est analysé. Quelquefois, nous apportons en cours d'exploitation des modifications aux expositions pour mieux répondre à la demande de nos publics.

Sur un plan personnel, quelle est votre exposition itinérante préférée et pourquoi ?

33 J'ai un faible pour InvenTerre, regards sur un vaisseau planétaire. C'est une exposition «art/sciences» dont la thématique est l'observation de la Terre et qui traite plus précisément des interactions entre les activités humaines et l'environnement. Il y a beaucoup d'expositions qui surfent sur cette thématique, mais InvenTerre propose un regard vraiment différent en offrant un voyage surprenant grâce aux satellites d'observation. Afin de compléter cette exposition, nous avons signé en 2009 un partenariat avec le Festival international de l'Image environnementale. L'exposition offre donc aujourd'hui deux regards : celui des satellites d'observation à travers quatre grands domaines de découvertes et celui de photographes professionnels "globe trotter » qui nous ont rapporté leurs visions de la fragilité de la Terre. 

exposition qui à mon sens est très équilibrée et susceptible d'interpeller un très large public. contenus de l'exposition. Le travail de conception fut très agréable, les relations avec Michel Lefevre, océanographe, passionnantes. En résumé, que de bons souvenirs !

Pour conclure, comment voyez-vous l'évolution du secteur des expositions itinérantes dans les années à venir?

Une multiplication de thèmes à tendances sociétales, des scénographies plus immersives avec de nouvelles technologies de moins en moins compliquées et de plus en plus étonnantes. De la 3D omniprésente. Un habillage plus émotionnel du contenu. Des nouveaux concepts « d'expositions à vivre " pour que la visite soit un véritable moment de découvertes et de plaisirs partagés.

\section{NOTES}

1. Le tarif moyen pour la location d'une exposition produite par la Cité de l'Espace est de 10000 euros HT/mois. Pour plus de renseignements : téléphone + 33562714876 ou + 33609996800 ; l.cossenet@cite-espace.com.

\section{RÉSUMÉS}

De Tunis à Marseille, de Copenhague à Montréal, chaque année, la Cité de l'Espace de Toulouse fait voyager ses expositions partout dans le monde. Avec un service spécialement dévolu à l'itinérance, l'établissement toulousain a ainsi développé un véritable savoir-faire en la matière. Responsable de cette activité au sein de l'institution, Laurent cossenet nous en explique les grands principes, ainsi que les objectifs.

\section{INDEX}

Mots-clés : Cité de l'Espace, exposition itinérante Index géographique : France, Toulouse 
AUTEUR

OLIVIER SOICHOT

Olivier Soichot est chargé d'édition à l'OCIM. olivier.soichot@u-bourgogne.fr 